\title{
Genetic Analysis of Disheveled 2 and Disheveled 3 in Human Neural Tube Defects
}

\author{
Patrizia De Marco • Elisa Merello • \\ Alessandro Consales • Gianluca Piatelli • \\ Armando Cama $\cdot$ Zoha Kibar • Valeria Capra
}

Received: 26 June 2012 / Accepted: 31 July 2012 /Published online: 15 August 2012

(C) Springer Science+Business Media, LLC 2012

\begin{abstract}
Neural tube defects are severe malformations affecting 1/1,000 live births. The planar cell polarity pathway controls the neural tube closure and has been implicated in the pathogenesis of neural tube defects both in animal models and human cohorts. In mouse disruption of $D v l 2$ alone $\left(D v l 2^{-/-}\right)$or $D v l 2$ and $D v l 3\left(D v l 2^{-/-} ; D v l 3^{+/-}, D v l 2^{+-}\right.$; $D v l 3^{-/-}$) results in incomplete neurulation, suggesting a role for Disheveled in neural tube closure. Disheveled is a multifunctional protein that is involved in both the canonical Wnt signaling and the noncanonical planar cell polarity pathway. In this study, we analyzed the role of the human orthologs DVL2 and DVL3 in a cohort of 473 patients with neural tube defects. Rare variants were genotyped in 639 ethnically matched controls. We identified seven rare missense mutations that were absent in all controls analyzed. Two of these mutations, p.Tyr667Cys and p.Ala53Val, identified in DVL2 were predicted to be detrimental in silico. Significantly, a 1-bp insertion (c.1801_1802insG) in exon 15 of $D V L 2$ predicted to lead to the truncation of the protein was identified in a patient with a complex form of caudal agenesis. In summary, we demonstrate a possible role for rare variants in $D V L 2$ gene as risk factors for neural tube defects.
\end{abstract}

Patrizia De Marco and Elisa Merello contribute equally to this work.

Electronic supplementary material The online version of this article (doi:10.1007/s12031-012-9871-9) contains supplementary material, which is available to authorized users.

P. De Marco • E. Merello • A. Consales · G. Piatelli · A. Cama •

V. Capra $(\bowtie)$

Neurosurgery Department, G. Gaslini Institute,

Genova 16148, Italy

e-mail: laboratorioNCH@ospedale-gaslini.ge.it

\section{Z. Kibar}

Department of Obstetrics and Gynecology, CHU Sainte Justine Research Center and University of Montreal,

Montreal, Québec H3T 1C5, Canada
Keywords Neural tube defects (NTDs) · Planar cell polarity (PCP) pathway $\cdot$ Mouse models $\cdot$ Disheveled (Dvl)

\section{Introduction}

Neural tube defects (NTDs; OMIM 182940) are a group of birth anomalies resulting from failure of fusion of the neural tube around the 28th day after conception. NTDs are known to occur in 1 out every 1,000 pregnancies in the USA, with varying rates reported among the world's populations (Botto et al. 1999; Kibar et al. 2007a). The most common NTDs are anencephaly, which results from failure of fusion of the cranial neural tube and myelomeningocele (commonly called spina bifida), which results from the failure of fusion of the neural tube in the spinal region. Failure of closure that involves the entire body axis is known as craniorachischisis that is an additional, lethal, and relatively rare form of dysraphism. Anencephaly and myelomeningocele are referred as "open" NTDs because the affected region is exposed to the body surface. There are also a number of closed or skin-covered conditions that involve the neural tube, including encephalocele, meningocele, lipomeningocele, also referred to as spina bifida occulta, and sacral agenesis (Rossi et al. 2004).

NTDs have a multifactorial etiology involving both environmental and genetic factors. Although folic acid, supplemented in the mother's diet periconceptionally, appears to have dramatically reduced the frequency of NTDs (MRC Vitamin Study Research Group 1991; Czeizel and Dudás 1992), the mechanism by which folate deficiency predisposes to NTDs remains unclear.

Consistent efforts in many research fields have focused toward understanding the mechanisms underlying the etiology of these devastating defects. Nevertheless, few candidate genes have proved to contribute to the development of 
NTDs. Of the genes that have been associated with NTDs, planar cell polarity (PCP) genes have been implicated as predisposing factors of isolated nonsyndromic NTDs (De Marco et al. 2011). The PCP pathway, a highly conserved, noncanonical Wnt-frizzled-disheveled signaling cascade, plays a key role in establishing and maintaining polarity in the plane of epithelia in Drosophila and in epithelial and nonepithelial tissues in vertebrates (Strutt 2003; Klein and Mlodzik 2005). Genetic studies of mutants affecting complex structures in the fly have identified a group of proteins referred to as "core" PCP components, that include transmembrane proteins such as Frizzled (Fz), Strabismus/Van Gogh (Stbm/Vang), and Flamingo (Fmi), as well as cytoplasmic proteins, including Disheveled (Dsh/Dvl), Prickle (Pk), and Diego (Dgo) (Jenny and Mlodzik 2006; Jones and Chen 2007).

The multifunctional protein Disheveled (Dsh in fly, XDsh in Xenopus, and Dvl1, Dvl2 and Dvl3 in mammals) is involved in both the canonical Wnt signaling pathway as well as the PCP pathway (Mlodzik 2002; Strutt 2002; Tree et al. 2002a; Wallingford and Harland 2002; Veeman et al. 2003a; Wallingford and Habas 2005) and regulates many biological processes, ranging from cell-fate specification and cell polarity to social behavior. In the canonical Wnt pathway, Dsh is important in transmitting a signal initiated from Wnt binding to its receptor Frizzled and coreceptor Arrow/Lrp5/6 on the cell surface. As a consequence, the cytoplasmic protein Armadillo/ $\beta$-catenin becomes stabilized and is transported into the nucleus to activate transcription of target genes (He et al. 2004; Wang and Wynshaw-Boris 2004). In the PCP pathway in the fly, Dsh participates with a different set of proteins such as Strabismus (Stbm), Prickle (Pk), Diego (Dgo), and Flamingo (Fmi) (Das et al. 2002; Jenny et al. 2005; Strutt 2002; Tree et al. 2002b; Veeman et al. 2003b). Although the precise mechanisms by which Dsh determines PCP signaling is not well defined, an asymmetric plasma membrane localization of Dsh and the other PCP members appears to be required for proper signaling of the PCP pathway in the fly (Axerold 2001; Strutt 2002; Tree et al. 2002a, b; Bastock et al. 2003; Jenny et al. 2003, 2005; Rawls and Wolff 2003; Veeman et al. 2003a; Das et al. 2002). Downstream effectors of Disheveled in the PCP pathway include the forminghomology protein DAAM1, two small GTPases, RhoA and Rac, and the kinases ROCK and JNK (Habas et al. 2001, 2003; Kim and Han 2005). All these proteins mediate cytoskeletal reorganization to induce cellular polarization, directed migration, and protrusive activity in migrating cells.

Disheveled proteins are 500-600 amino acids in length and are modular. They function as essential scaffold proteins that interact with diverse proteins, including kinases, phosphatases, and adaptor proteins. They contain three highly conserved domains: the DIX (Disheveled/Axin) domain that is largely $\alpha$-helical structure (Capelluto et al. 2002), the PDZ (PSD-95, DLG,ZO1) domain, which consists of six $\beta$-sheets that enfold two $\alpha$-helices (Wong et al. 2003), and the DEP (Disheveled, EGL-10, Pleckstrin) domain, consisting of a bundle of three $\alpha$-helices domain (Wong et al. 2000). Several additional conserved regions are considered critical for biological function: the serine/threonine stretches between the DIX and PDZ domains, and the proline-rich regions with a $\mathrm{SH} 3$ protein-binding domain motif downstream of the PDZ. The central PDZ and the DEP domains function in noncanonical PCP signaling.

Mutants of Disheveled which correspond to mutations in Drosophila that selectively impair PCP signaling were found to disrupt convergent extension when expressed in Xenopus (Wallingford et al. 2000) or zebrafish (Heisenberg et al. 2000). In mouse, $D v l 1^{--}, D v l 3^{--}$single mutants and $D v l 1^{-/-} ; D v l 3^{-1}$ - double mutants do not display neural tube defects (Lijam et al. 1997; Wang et al. 2006). $D v l 2^{-/-}$embryos displayed thoracic spina bifida, while virtually all $D v l 1^{--} ; D v l 2^{--}$double mutant embryos displayed craniorachishisis, a completely open neural tube from the midbrain to the tail (Hamblet et al. 2002; Wang et al. 2006). For $D v l 3$, neurulation appeared normal both in $\mathrm{Dvl}^{-/-}$and $\operatorname{Ltap}^{\mathrm{Lp/+}}$ (Vangl2/Ltap) mutants, while defects were seen in both $\mathrm{Dvl3^{+/- }} ; \mathrm{Ltap}^{\mathrm{Lp} /+}$ and $\mathrm{Dvl3^{-/- }}$; $L_{t a p}{ }^{L p /+}$ mutants, indicating genetic interaction between the $D v l$ genes and the other PCP gene Vangl2 (Etheridge et al. 2008). These findings indicate that $D v l 2$ is the most important mammalian $D v l$ gene for neural tube closure and is sufficient by itself for the correct process. By contrast, $D v l l$ and $D v l 3$ are not sufficient by themselves but contribute significantly when Dvl2 is completely missing (Wu et al. 2011).

In view of these data and given the implication of PCP signaling in NTDs, we investigated if the human orthologs DVL2 and DVL3 genes could play a role in NTDs pathogenesis.

\section{Patients and Methods}

The patient cohort consisted of (a) 391 Italian patients recruited at the Spina Bifida Center of the Gaslini Hospital in Genova, Italy; (b) 82 patients (mainly French-Canadian) recruited at Sainte Justine Hospital in Montreal, Canada. Both groups were already included in our previous studies (Kibar et al. 2007b, 2009, 2011; De Marco et al. 2012). All patients had isolated nonsyndromic NTDs. Around $19 \%$ of patients had a positive family history documented by clinical records (MRI and X-ray images).

The control group comprised 433 Italian individuals and 206 Caucasian controls of French-Canadian ancestry previously enrolled for our genotyping study (De Marco et al. 2012). All control individuals $(N=639)$ were seen by a 
neurologist and confirmed to be healthy. Samples from patients and controls were collected with the approval of the Local Ethics Committees, and written informed consent was obtained from all participating patients, parents, and controls.

\section{Resequencing and Genotyping}

Genomic DNA was isolated from EDTA peripheral blood samples, by using the QIAamp DNA blood Kit (Qiagen), according to the manufacturer's protocol. The genomic structures of human $D V L 2$ and $D V L 3$ were determined using the NCBI GenBank (DVL2, NM_004422.2; DVL3, NM_004423.3). Primers flanking the exon-intron junctions were developed manually. The whole coding region of DVL2 (3,046-bp long) and DVL3 (5,062-bp long) with $180 \mathrm{bp}$ of the $5^{\prime}$-untranslated region (UTR) and $150 \mathrm{bp}$ of the 3'UTR was amplified in a total of 10-12 amplicons for each gene. The portions of the introns that were sequenced ranged from 120 to $160 \mathrm{bp}$ with an average of almost 140 bp. Polymerase chain reaction (PCR) was carried out using the AmpliTaqGold (Applied Biosystems) as per manufacturer's instructions. Direct dye terminator sequencing of PCR products was carried out using the ABI Prism Big Dye Systems (Applied Biosystems). Samples were run on ABI 3700 automated sequencer and analyzed using the PhredPhrap software 5.04 (http://droog.gs.washington.edu/ polyphred). Nonsynonymous changes that were most likely expected to have a detrimental effect were further genotyped using the iPLEX ${ }^{\mathrm{TM}}$ Gold assay for SNP Genotyping (Sequenom, San Diego, CA) (Ehrich et al. 2005). For variants identified in NTDs cases, we tested the cosegregation by sequencing the corresponding fragment in available additional family members.

\section{Bioinformatics}

Mutations were annotated according to the HGVS nomenclature (http://www.hgvs.org/mutnomen). Nucleotide numbering reflects cDNA numbering with +1 corresponding to the A of the ATG translation initiation codon 1 in the reference sequence. All sequence variations were queried against three public databases: the dbSNP Build 133, the 1,000 Genome Project (release of May 2011), and the NHLBI GO Exome Sequencing Project (http://snp.gs.washington.edu/EVS/). To predict the likelihood that an amino acid change is deleterious, we used two software programs: PolyPhen (polymorphism phenotyping) (http://genetics.bwh.harvard.edu/pph/) and SIFT (Sorting Intolerant from Tolerant software). Default conditions were used for the programs. Nonfunctional rare variants have been checked for creation of cryptic splice sites using computational method alternative splice site predictor (http://www.es.embnet.org/ mwang/assp.html). Multiple alignments of the DVL2 and DVL3 proteins were done using the CLUSTAL W program, freely available online (http:// npsa-pbil.ibcp.fr). Localization of the variants in protein domains were assessed by Uniprot (http://www.uniprot.org/).

Potential effect of the $3^{\prime}$ UTR variants by altering MicroRNA (miRNA) binding sites was evaluated by MicroRNA.org site (http://www.microrna.org) that uses the mirSVR regression model for target site predictions (mirSVR score $\leq-0.1$ ).

\section{Association Analysis}

Differences in mutation burden between patients and controls were assessed by two-sided $t$ tests, using SPSS version 16.0.2 (Brussels, Belgium).

\section{Results}

We report a genetic molecular study of DVL2 and DVL3 by resequencing a large cohort of 473 NTD patients to test the hypothesis whether a defective PCP signaling resulting from mutations in $D V L$ genes could represent a common underlying pathogenic mechanism.

We detected a total of eight rare mutations $(<1 \%)$ in patients, five in $D V L 2$ and three in $D V L 3$, that were not present in all controls analyzed. With the exception of $D V L 2$ p.Ala53 Val, all of them were novel and not reported in the public databases (Table 1). All these mutations were found in a heterozygote state, and all were private. They included a 1-bp insertion (c.1801_1802insG) in exon 15 of DVL2 predicted to lead to a premature truncation of the protein (p.Glu620X) and seven missense mutations mainly localized in the C-terminal of the protein. The 1-bp insertion in exon 15 of $D V L 2$ was detected in a girl affected by caudal agenesis and tethered cord. Parents were not available for the genetic testing. The mutation introduces a premature stop codon at position 620 and truncated the protein that lacked the last 116 amino acids in its carboxyl-terminal tail. The majority of the missense mutations affected conserved amino acid residues among DVLs orthologs (data not shown). Two out seven missense mutations (DVL2 p.Tyr667Cys and DVL2 p.Ala53 Val) were predicted to be damaging or having functional relevance according to the SIFT program. The two missense changes of DVL2, p.Tyr667Cys and p.Ala53Val, were found in two sporadic cases of NTDs, having myelomeningocele and lipoma, respectively. Interestingly, the p.Ala53Val affects the aminoterminal DIX domain which mediates dynamic polymerization, essential for signaling activity of DVL2 (SchwarzRomond et al. 2007). The DVL2 p.Ala53Val (rs149736410) was detected twice among 2,500 exomes included in the NHLBI Exome Sequencing Project at a frequency of 0.001 . 
Table 1 Novel rare mutations $(<1 \%)$ identified in DVL2 and $D V L 3$ in human cohorts

\begin{tabular}{|c|c|c|c|c|c|c|}
\hline Mutation & AA change & $\begin{array}{l}\text { Patients } \\
\text { (473) }\end{array}$ & $\begin{array}{l}\text { Controls }{ }^{\mathrm{a}} \\
(150)\end{array}$ & Polyphen ${ }^{\mathrm{b}}$ & $\mathrm{SIFT}^{\mathrm{c}}$ & Domain \\
\hline \multicolumn{7}{|l|}{$D V L 2$ rare mutations } \\
\hline \multicolumn{7}{|c|}{ Potentially damaging variants } \\
\hline c. $158 \mathrm{C}>\mathrm{T}$ & p.Ala53 Val ${ }^{\mathrm{d}}$ & 1 & 0 & - & + & DIX \\
\hline c. $1073 \mathrm{C}>\mathrm{T}$ & p.Ser358Phe & 1 & 1 & + & + & Proline-rich region \\
\hline c.1801_1802insG & p.Glu620X & 1 & 0 & N/A & $\mathrm{N} / \mathrm{A}$ & C-terminal \\
\hline c. $2000 \mathrm{~A}>\mathrm{G}$ & p.Tyr667Cys & 1 & 0 & - & + & C-terminal \\
\hline \multicolumn{7}{|l|}{ Benign variants } \\
\hline c. $332 \mathrm{C}>\mathrm{T}$ & p.Ala111Val & 1 & 0 & - & - & Polypeptide linker \\
\hline c. $1786 \mathrm{C}>\mathrm{T}$ & p.Arg596Trp & 1 & 0 & - & - & C-terminal \\
\hline Total & & 6 & 1 & & & \\
\hline \multicolumn{7}{|l|}{ DVL3 rare mutations } \\
\hline \multicolumn{7}{|c|}{ Potentially damaging variants } \\
\hline c. $523 \mathrm{~A}>\mathrm{G}$ & p.Ser175Gly ${ }^{\mathrm{e}}$ & 0 & 1 & ++ & + & Polypeptide linker \\
\hline \multicolumn{7}{|l|}{ Benign variants } \\
\hline c. $1057 \mathrm{~A}>\mathrm{G}$ & p.Ile353Val & 1 & 0 & - & - & Polypeptide linker \\
\hline c. $1147 \mathrm{~A}>\mathrm{G}$ & p.Ile384Val & 1 & 0 & - & - & Polypeptide linker \\
\hline c. $1921 \mathrm{G}>\mathrm{A}$ & p.Ala641Thr & 1 & 0 & - & - & C-terminal \\
\hline Total & & 3 & 1 & & & \\
\hline$D V L 2+D V L 3$ variants & & 9 & 2 & & & \\
\hline
\end{tabular}

$A A$ amino acid, Polyphen polymorphism phenotyping software, SIFT Sorting Intolerant from Tolerant software

${ }^{a}$ All the nonsynonymous variants identified in patients were genotyped in 639 controls

${ }^{\mathrm{b}}$ Polyphen predictions for amino acid changes: "++" for probably damaging "+" for possibly damaging; "-" for benign

'SIFT predictions for amino acid changes: "+" predicted to affect protein function; "-" predicted to be tolerated

${ }^{\mathrm{d}}$ The variant is present in database as rs149736410

${ }^{\mathrm{e}}$ The variant is present in database as rs149103009
None of the three DVL3 missense variants detected in patients and absent in controls caused significant changes in the protein function since they were scored as neutral by the two algorithms. Moreover, these mutations did not create cryptic splice sites, suggesting that they did not affect the gene splicing.

A single nucleotide substitution (c.*49 $\mathrm{G}>\mathrm{C}$ ) affecting the $3^{\prime}$ UTR of the DVL3 gene, outside of protein coding sequence, was also identified in one patient (data not shown). Potential effect of this novel rare variant by altering miRNA binding sites was evaluated, but no evident effect was demonstrated. Detailed clinical information on patients carrying rare mutations of $D V L s$ are presented in Supplementary Table 1.

To assess the rate of rare pathogenic mutations in $D V L 2$ and $D V L 3$ in the normal population, we therefore resequenced the coding sequence and exon-intron boundaries of the two genes in a cohort of 150 ethnically matched controls. We could detect one missense mutation in DVL2, p. Ser358Phe, and one in DVL3, p. Ser175Gly, in two healthy individuals. The $D V L 2 \mathrm{p}$. Ser358Phe and the DVL3 p. Ser175Gly gene variants were predicted to affect protein function in silico (Table 1). There was no significant difference in overall mutation burden between the genotyped patients (nine nonsynonymous rare variants/473) and controls (two nonsynonymous rare variants/150). Nevertheless, when only $D V L 2$ variants were taken into account, the overall rate of predicted deleterious variants causing amino acid substitutions (including frameshifts) were 1.2-fold higher in cases compared to controls (4/473 in patients $v s 1 / 150$ in controls), although this trend did not reach the significance $(p=1)$.

In addition to these rare mutations, we found a common variant, the $D V L 2$ p.Thr535Ile (rs72839768), at a frequency of $1 \%$ in patient group. This variant was not conserved and was predicted to be damaging; nevertheless, an association study between case and control groups demonstrated no significant association of this SNP with NTD phenotype (data not shown).

The patient group belongs to a cohort already analyzed for mutations in other PCP genes VANGL1, VANGL2, PRICKLE1, CELSR1, FZD3, and FZD6 (Kibar et al. 2009, 2011; Bosoi et al. 2011; Allache et al. 2012; De Marco et al. 2012). While no NTD patient and control was a carrier of rare missense mutations in both $D V L 2$ and $D V L 3$, we could identify four patients that were double heterozygotes for variants in one of the $D V L$ genes and in another core PCP gene (Table 2).

\section{Discussion}

Disheveled (DVL) proteins are important signaling components of both the canonical Wnt signaling pathway, which 
coordinates cell proliferation and patterning, and the PCP pathway, which coordinates cell polarity within a sheet of cells and also directs convergent extension movements and neurulation in vertebrates. Evidence for the involvement of the DVLs genes as well as others core PCP genes in neurulation have emerged from studies of a wide range of mutants of orthologs of PCP genes in several animal models. Recently, we and other groups have demonstrated the link between some of the PCP genes, such as VANGL1, VANGL2, PRICKLE1, CELSR1, and FZD6, and human NTDs (Kibar et al. 2007b, 2009, 2011; Bosoi et al. 2011; Allache et al. 2012; De Marco et al. 2012).

In this study, we analyzed the role of DVL2 and DVL3 in a large group of 473 NTD patients and 150 controls to determine whether mutations at these genes predispose to these birth defects. Prediction and understanding of the downstream effects of the nonsynonymous variants was done using computational methods.

An overall low mutation burden both in patients and controls was found probably due to the pivotal role of DVL proteins in biological processes, so that highly damaging mutations or changing in critical and conserved positions essential for the structure and/or function of the protein are not tolerated. We suggested that mutations at fully conserved sites of DVL proteins may be lethal, thus accounting for their relative absence in data sets, while those at moderately conserved sites are more likely to produce the observed disease phenotype in patients.

Nevertheless, we identified a total of seven rare (MAF $>1 \%)$ missense mutations in seven patients that were uniquely present in patients and absent in all controls analyzed and two of them, p.Tyr667Cys and p.Ala53 Val, in DVL2 were predicted to be detrimental in silico and were detected in two sporadic cases of NTDs. Importantly, we also identified a 1-bp insertion (c.1801_1802insG) in exon 15 of $D V L 2$ predicted to lead to a premature truncation (p.Glu620X) of the protein that lacks its C-terminal tail. Although the impact of this variant will need to be evaluated, it is probably that the truncated form of the protein could affect intracellular interactions or alternatively could play a dominant negative role by inhibiting the wild-type protein. Genotype-phenotype correlation shows that the DVL2

Table 2 Patients carrying variants both in $D V L$ s genes and in a second core PCP gene

\begin{tabular}{lll}
\hline DVL variant & PCP gene variants & Clinical phenotype \\
\hline DVL2 p.Thr535Ile & VANGL2 p. Arg135Trp & MMC \\
DVL2 p. Glu620X & VANGL2 p. Arg482His & CA \\
DVL2 p. Ala111Val & CELSR1 p.Thr346Ser & Lipoma \\
DVL3 p. Ile353Val & VANGL1 p. Ala404Ser & CA \\
\hline
\end{tabular}

${ }^{\mathrm{a}}$ Kibar et al. 2011; ${ }^{\mathrm{b}}$ Allache et al. 2012; ${ }^{\mathrm{c}}$ Kibar et al. 2009 frame-shift mutation is associated with a complex dysraphism in a patient presenting with caudal agenesis and tethered cord.

The identification of rare functional variants in DVL2 suggests an independent role of this gene in the pathogenesis of a minority of NTD patients and underscores the value of candidate gene resequencing to understand the genetic contribution in these birth defects. However, the absence of a statistically significant burden of rare pathogenic mutations of DVL2 in cases versus controls in our population may be attributed to the occurrence of rare variants, having frequencies too low to be able to run adequate statistical comparisons. A much larger case-control mutation burden analysis would be needed to answer this important question.

All the pathogenic DVL2 mutations were identified in sporadic cases without reported family history and were private. When the parents were available, the mutation was inherited from an unaffected parent, demonstrating incomplete penetrance of the mutations.

Our findings suggest that $D V L 2$ gene could contribute in the pathogenesis of both open and closed forms of NTDs, as reported for other PCP genes (Kibar et al. 2007b Kibar et al. 2009; Kibar et al. 2011), further supporting a common mechanism involving defective PCP genes in the onset of both NTD forms.

By contrast, we could not demonstrate a significant contribution of $D V L 3$ gene in the pathogenesis of NTDs. In fact, although the p.Ile353Val and p.Ile384Val missense variants affect residues conserved across the evolution, both mutations did not have an evident pathogenic effect on protein function. Further studies would be required to determine whether DVL3 may play an independent role in the development of NTDs.

A predominant role of $D V L 2$ over $D V L 3$ is in agreement with mouse models demonstrating that $D v l 2$ is the most important gene to promote neural tube closure and is sufficient by itself for correct closure. In fact, $D v l l$ and $D v l 3$ are not sufficient by themselves but contribute significantly to neural tube closure only when $D v l 2$ is completely missing (Wang et al. 2006). The discrepancy between human and mouse models is represented by the phenotype resulting from gene inactivation; differently from humans where we found DVL2 mutations mainly in closed forms of NTDs, knockouts in $D v l 2$ gene have been implicated only in open forms of NTDs including spina bifida and craniorachischisis. In general, the role of a defective PCP signaling in closed forms of NTDs in mouse models has not been investigated yet.

Interestingly, we provide evidences of digenic occurrence of mutations at two core PCP genes, one of the two DVL genes and a second core PCP gene. In fact, we identified two patients that were double heterozygote for missense variants in DVL2 and VANGL2 (DVL2 p.Thr535Ile/ 
VANGL2 p.Arg135Trp; DVL2 p. Glu620X/VANGL2 p.Arg482His), one patient in DVL2 and CELSR1 (DVL2 p.Ala111Val/CELSR1 p. Thr346Ser), and last one in DVL3 and VANGL1 (DVL3 p.Ile353Val/VANGL1 p.Ala404Ser). Genetic interactions between core PCP genes have been demonstrated in double mutant mice developing alteration of neural tissue closure processes, including both open rostral and caudal neural tube defects (Harris and Juriloff 2010; Yu et al. 2010). According to the multifactorial model of human NTD etiology, here we report that human $D V L$ mutations may genetically interact with $V A N G L$ and CELSR1 alleles to affect neural tube closure, although we could not exclude that two mutations in the same individual may be a coincidental finding. Replication of our findings in larger sample sets will, however, be required to further substantiate the observed effects.

In conclusion, to our knowledge, this study is the first describing a comprehensive resequencing analysis of the $D V L$ genes in NTDs. Our findings support the involvement of rare DVL2 variants in NTD etiology and emphasize the role of PCP genes in neural tube closure. Moreover, potential genetic interactions of mutations in more than one PCP gene represent a further step forward to our understanding of the intricate genetic puzzle underlying these complex malformations. Further screening of DVL3 gene and genetic analysis of DVL1 is warranted. Finally, functional studies of the $D V L$ variants are needed to confirm their implication in NTDs risk.

Acknowledgments We are grateful to all patients and their families. This work was supported by Italian Telethon Foundation (grant no. GGP08051) and by the Canadian Institutes for Health Research, the Sick Kids Foundation, the Fonds de la Recherche en Santé du Québec. We also thank A.S.B.I. (Associazione Spina Bifida Italia), Ruiu Ilaria, and Mara Uglietta for technical assistance.

\section{References}

Allache R, De Marco P, Merello E, Capra V, Kibar Z (2012) Role of the planar cell polarity gene CELSR1 in neural tube defects and caudal agenesis. Birth Defects Res A Clin Mol Teratol 94 (3): $176-181$

Axerold JD (2001) Unipolar membrane association of dishevelled mediates Frizzled planar cell polarity signalling. Genes Dev $15: 1182-1187$

Bastock R, Strutt H, Strutt D (2003) Strabismus is asymmetrically localised and binds to Prickle and Dishevelled during Drosophila planar polarity patterning. Development 130:3007-3014

Bosoi CM, Capra V, Trinh VQH, De Marco P, Merello E, Drapeau P, Bassuk AG, Kibar Z (2011) Identification and characterization of novel rare mutations in the planar cell polarity gene PRICKLE1 in human neural tube defects. Hum Mutat 32(12):1371-1375

Botto LD, Moore CA, Khoury MJ, Erickson JD (1999) Neural-tube defects. N Engl J Med 341:1509-1519
Capelluto DG, Kutateladze TG, Habas R, Finkielstein CV, He X, Overduin M (2002) The DIX domain targets dishevelled to actin stress fibres and vesicular membranes. Nature 419:726-729

Czeizel AE, Dudás I (1992) Prevention of the first occurrence of neural-tube defects by periconceptional vitamin supplementation. N Engl J Med 327:1832-1835

Das G, Reynolds-Kenneally J, Mlodzik M (2002) The atypical cadherin Flamingo links Frizzled and Notch signaling in planar polarity establishment in the Drosophila eye. Dev Cell 2:655-666

De Marco P, Merello E, Capra V (2011) Neural tube defects: genetic causes and prevention. Biofactor 37(4):261-268

De Marco P, Merello E, Rossi A, Piatelli G, Cama A, Kibar Z, Capra V (2012) FZD6 is a novel gene for human neural tube defects. Hum Mutat 33(2):384-390

Ehrich M, Bocker S, Van den Boom D (2005) Multiplexed discovery of sequence polymorphism using base-specific cleavage and MALDI-TOF MS. Nucleic Acids Res 33:e38

Etheridge SL, Ray S, Li S, Hamblet NS, Lijam N, Tsang M, Greer J, Kardios N, Wang J, Sussman DJ, Chen P, Wynshaw-Boris A (2008) Murine dishevelled 3 functions in redundant pathways with dishevelled 1 and 2 in normal cardiac outflow tract, cochlea, and neural tube development. PLoS Genet 4(11):e1000259

Habas R, Kato Y, He X (2001) Wnt/Frizzled activation of Rho regulates vertebrate gastrulation and requires a novel Formin homology protein Daam1. Cell 107:843-854

Habas R, Dawid IB, He X (2003) Coactivation of Rac and Rho by Wnt/Frizzled signalling is required for vertebrate gastrulation. Genes Dev 17:295-309

Hamblet NS, Lijam N, Ruiz-Lozano P, Wang J, Yang Y, Luo Z, Mei L, Chien KR, Sussman DJ, Wynshaw-Boris A (2002) Dishevelled 2 is essential for cardiac outflow tract development, somite segmentation and neural tube closure. Development 129:5827-5838

Harris MJ, Juriloff DM (2010) An update to the list of mouse mutants with neural tube closure defects and advances toward a complete genetic perspective of neural tube closure. Birth Defects Res A Clin Mol Teratol 88(8):653-669

He X, Semenov M, Tamai K, Zeng X (2004) LDL receptor-related proteins 5 and 6 in $\mathrm{Wnt} / \beta$-catenin signalling: arrows point the way. Development 131:1663-1677

Heisenberg CP, Tada M, Rauch GJ, Saude L, Concha ML, Geisler R, Stemple DL, Smith JC, Wilson SW (2000) Silberblick/Wnt11 mediates convergent extension movements during zebrafish gastrulation. Nature 405:76-81

Jenny A, Mlodzik M (2006) Planar cell polarity signaling: a common mechanism for cellular polarization. Mt Sinai J Med 73:738-750

Jenny A, Darken RS, Wilson PA, Mlodzik M (2003) Prickle and Strabismus form a functional complex to generate a correct axis during planar cell polarity signaling. EMBO J 22:4409-4420

Jenny A, Reynolds-Kenneally J, Das G, Burnett M, Mlodzik M (2005) Diego and Prickle regulate Frizzled planar cell polarity signalling by competing for Dishevelled binding. Nat Cell Biol 7:691-697

Jones C, Chen P (2007) Planar cell polarity signaling in vertebrates. Bioessays 29(2):120-132

Kibar Z, Capra V, Gros P (2007a) Toward understanding the genetic basis of neural tube defects. Clin Genet 71(4):295-310

Kibar Z, Torban E, Mc Dearmid JR, Reynolds A, Mathieu M, Kirillova I, De Marco P, Merello E, Hayes JM, Wallingford JB, Drapeau P, Capra V, Gros P (2007b) Mutations in VANGL1 associated in neural-tube defects. N Engl J Med 361(14):1432-1437

Kibar Z, Bosoi CM, Kooistra M, Salem S, Finnell RH, De Marco P, Merello E, Bassuk AG, Capra V, Gros P (2009) Novel mutations in VANGL1 in neural tube defects. Hum Mutat 30:E706-E715

Kibar Z, Salem S, Bosoi CM, Pauwels E, De Marco P, Merello E, Bassuk AG, Capra V, Gros P (2011) Contribution of VANGL2 mutations to isolated neural tube defects. Clin Genet 80(1):76-82 
Kim GH, Han JK (2005) JNK and ROK $\alpha$ function in the noncanonical Wnt/RhoA signalling pathway to regulate Xenopus convergent extension movements. Dev Dyn 232:958-968

Klein TJ, Mlodzik M (2005) Planar cell polarization: an emerging model points in the right direction. Annu Cell Dev Biol 21:155176

Lijam N, Paylor R, McDonald MP, Crawley JN, Deng CX, Herrup K, Stevens KE, Maccaferri G, McBain CJ, Sussman DJ, WynshawBoris A (1997) Social interaction and sensorimotor gating abnormalities in mice lacking Dvl1. Cell 90:895-905

Mlodzik M (2002) Planar cell polarization: do the same nechanisms regulate Drosophila tissue polarity and vertebrate gastrulation? Trends Genet 18:564-571

MRC Vitamin Study Research Group (1991) Prevention of neural tube defects: results of the Medical Research Council Vitamin study. Lancet 338:131-137

Rawls AS, Wolff T (2003) Strabismus requires Flamingo and Prickle function to regulate tissue polarity in the Drosophila eye. Development 130(9):1877-1887

Rossi A, Cama A, Piatelli G, Ravegnani M, Biancheri R, TortoriDonati P (2004) Spinal dysraphism: MR imaging rationale. J Neuroradiol 31:3-24

Schwarz-Romond T, Fiedler M, Shibata N, Butler PJG, Kikuchi A, Higuchi Y, Bienz M (2007) The DIX domain of Dishevelled confers Wnt signaling by dynamic polymerization. Nat Struct Mol Biol 14(6):484-492

Strutt DI (2002) The asymmetric subcellular localisation of components of the planar polarity pathway. Semin Cell Dev Biol 13:225-231

Strutt D (2003) Frizzled signalling and cell polarisation in Drosophila and vertebrates. Development 130:4501-4513

Tree DR, Ma D, Axelrod JD (2002a) A three-tiered mechanism for regulation of planar cell polarity. Semin Cell Dev Biol 13:217224

Tree DR, Shulman JM, Rousset R, Scott MP, Gubb D, Axerold JD (2002b) Prickle mediates feedback amplification to generate asymmetric planar cell polarity signaling. Cell 109:371-381
Veeman MT, Axelrod JD, Moon RT (2003a) A second canon. Functions and mechanisms of beta-catenin-independent Wnt signaling. Dev Cell 5:367-377

Veeman MT, Slusarski DC, Kaykas A, Louie SH, Moon RT (2003b) Zebrafish prickle, a modulator of noncanonical Wnt/Fz signaling, regulates gastrulation movements. Curr Biol 13:680-685

Wallingford JB, Rowning BA, Vogeli KM, Rothbacher U, Fraser SE, Harland RM (2000) Dishevelled controls cell polarity during Xenopus gastrulation. Nature 405:81-85

Wallingford JB, Harland RM (2002) Neural tube closure requires Dishevelled-dependent convergent extension of the midline. Development 129:5815-5825

Wallingford JB, Habas R (2005) The developmental biology of Dishevelled: an enigmatic protein governing cell fate and cell polarity. Development 132:4421-4436

Wang J, Wynshaw-Boris A (2004) The canonical Wnt pathway in early mammalian embryogenesis and stem cell maintenance/differentiation. Curr Opin Genet Dev 14:533-539

Wang J, Hamblet NS, Mark S, Dickinson ME, Brinkman BC, Segil N, Fraser SE, Chen P, Wallingford JB, Wynshaw-Boris A (2006) Dishevelled genes mediate a conserved mammalian PCP pathway to regulate convergent extension during neurulation. Development $133: 1767-1778$

Wong HC, Mao J, Nguyen JT, Srinivas S, Zhang W, Liu B, Li L, Wu D, Zheng J (2000) Structural basis of the recognition of the Dishevelled DEP domain in the Wnt signalling pathway. Nat Struct Biol 7:1178-1184

Wong HC, Bourdelas A, Krauss A, Lee HJ, Shao Y, Wu D, Mlodzik M, Shi DL, Zheng J (2003) Direct binding of the PDZ domain of Dishevelled to a conserved internal sequence in the C-terminal region of Frizzled. Mol Cell 12:1251-1260

Wu G, Huang X, Hua Y, Mu D (2011) Roles of planar cell polarity pathways in the development of neural tube defects. J Biomed Sci 18:66

Yu H, Smallwood PM, Wang Y, Vidaltamayo R, Reed R, Nathans J (2010) Frizzled 1 and frizzled 2 genes function in palate, ventricular septum and neural tube closure: general implications for tissue fusion processes. Development 137:3707-3717 